\title{
« 2015, Année de la lumière en France » est un immense succès!
}

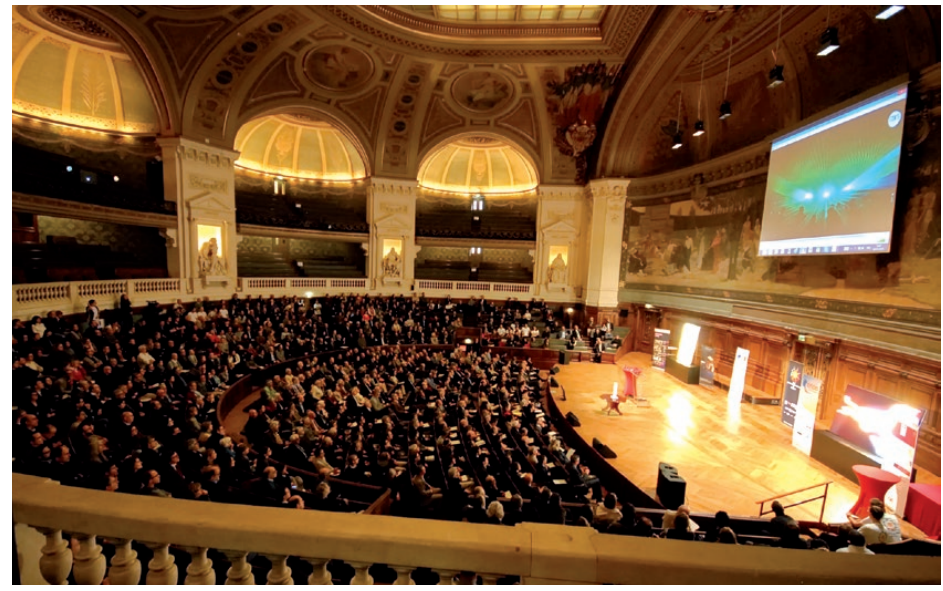

1. La cérémonie de lancement dans le grand amphithéâtre de la Sorbonne, le 8 janvier 2015.

En France, l'Année de la lumière s'est déroulée sous le haut patronage de Monsieur François Hollande, président de la République, et sous le parrainage de deux lauréats du prix Nobel de physique, Claude Cohen-Tannoudji et Serge Haroche.

Ouverte le 8 janvier, lors d'une prestigieuse manifestation de lancement dans le grand amphithéâtre de la Sorbonne (fig. 1), «2015, Année de la lumière en France " s'est révélée un grand succès populaire.

L'ensemble des régions se sont impliquées dans ces manifestations centrées sur la lumière, de Paris à toutes les métropoles régionales, des petites villes jusqu'aux villages, des salles de spectacles et amphithéâtres aux collèges, lycées et MJC, des festivals aux grandes conférences. Nos commissions ont labellisé à ce jour plus de 1000 évènements, dans tous les domaines : arts, culture, éducation, jeunesse, astronomie, éclairage, vision, recherche, industrie, santé.

Les manifestations sont et ont été majoritairement orientées vers les jeunes (fig. 2) et le grand public. Enseigner librement la lumière aux jeunes à partir de 7 ans, faciliter la diffusion des connaissances dans les écoles à destination des élèves mais aussi des enseignants et des parents, fut notre mission principale tout au long de cette année des lumières. Les jeunes ont eu ainsi l'occasion de découvrir toute l'étendue du potentiel des sciences et des techniques qui utilisent la lumière, ainsi que les opportunités en termes de retombées industrielles et d'emplois.
L'Organisation des Nations Unies a proclamé l'année 2015 Année internationale de la lumière et des techniques utilisant la lumière. L'ONU souhaite ainsi sensibiliser et mieux former la communauté internationale aux sciences et techniques de la lumière pour relever des défis tels que le développement durable, l'énergie et la santé des collectivités, et pour améliorer la qualité de vie tant dans les pays développés que dans les pays en développement. L'ONU considère que les applications des sciences et techniques de la lumière sont capitales pour les progrès actuels et futurs dans des domaines tels que la médecine, l'énergie, l'information et les communications, les fibres optiques, l'agriculture, les industries extractives, I'astronomie, I'architecture, I'archéologie, le divertissement, l'art et la culture.

La Cérémonie de clôture de « 2015, Année de la lumière en France ", a eu lieu le 23 février 2016 à Paris, ville lumière, dans les salons de l'Hôtel de Ville. La Mairie de Paris fut le partenaire de cette belle fête, qui s'est déroulée de 13 h 30 à 21 h 00 dans la grande salle des fêtes de la Mairie, le salon des Arcades et le salon Georges Bertrand.

Devant près de 700 invités se sont succédés une quinzaine d'intervenants, scientifiques et artistes, qui ont souligné la diversité des utilisations de la lumière et le potentiel extraordinaire qu'elle représente pour notre société.

Au cours de cette cérémonie, le prix Jean Jerphagnon 2015, qui récompense l'excellence en optique-photonique au coeur de l'entreprenariat, a été décerné à Frédéric Gérôme (Institut XLIM, Limoges), cofondateur de la société GLOphotonics.

Devant le nombre important de demandes d'organisation d'évènements au-delà du 31 décembre 2015, le Comité national de l'Année de la lumière en France a décidé de prolonger cette année exceptionnelle jusqu'au 30 juin 2016.

Une cérémonie festive de célébration est envisagée à Lyon à l'automne 2016. Elle permettra aux acteurs régionaux et nationaux qui auront contribué au succès de l'«Année de la lumière en France " de participer à un beau rassemblement à connotation artistique : spectacles de lumière, pièces de théâtre, animations visuelles.

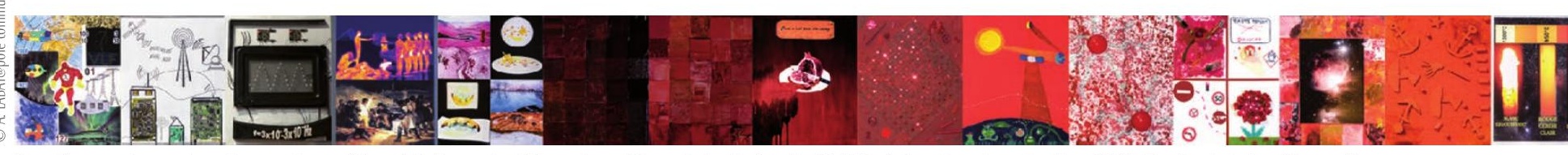

2. « Des couleurs dans le spectre », frise réalisée par les élèves des collèges et des lycées de Midi-Pyrénées dans le cadre de «2015, Année de la lumière en France ». 

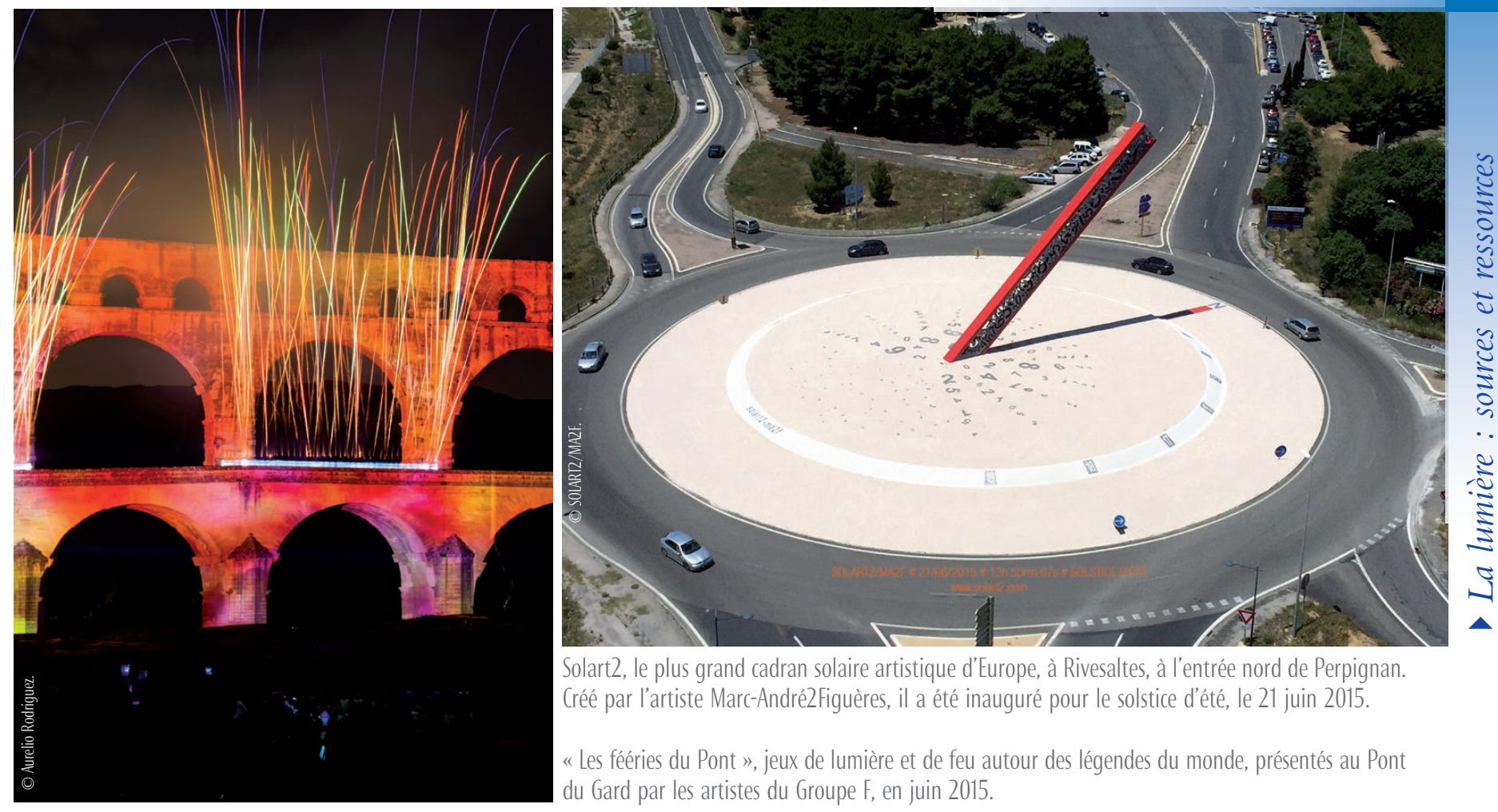

Solart2, le plus grand cadran solaire artistique d’Europe, à Rivesaltes, à l'entrée nord de Perpignan. Créé par l'artiste Marc-André2Figuères, il a été inauguré pour le solstice d’été, le 21 juin 2015.

"Les feéries du Pont », jeux de lumière et de feu autour des légendes du monde, présentés au Pont du Gard par les artistes du Groupe F, en juin 2015.

\section{Pourqụoi avoir choisi l'année 2015 pour célébrer la lumière?}

2015 correspond à plusieurs dates clés anniversaires dans le domaine scientifique, liées à l'optique et à la lumière.

Tout d'abord 1015, qui voit paraitre le premier Traité d'optique, ouvrage en sept volumes, écrit par Alhazen (nom latinisé d'Ibn al-Haytham). Le livre a eu une influence importante sur le développement de l'optique et de la science en général, car il a transformé radicalement la connaissance de la lumière et de la vision, et a introduit la méthode scientifique expérimentale. En 1815, Augustin Fresnel, physicien français, s'oppose à la théorie corpusculaire de la lumière de Newton en vigueur jusque-là et, par des expériences de diffraction, pose les bases de sa théorie "vibratoire " de la lumière. C'est en 1865 que Maxwell décrit la théorie électromagnétique dynamique de la lumière. En 1905, Einstein a interprété l'effet photoélectrique puis, en 1915, il a élaboré la théorie de la relativité générale, en montrant comment la lumière était au centre de la structure même de l'espace et du temps. Enfin, plus proche de nous, en 1965 Penzias et Wilson ont découvert le fond diffus cosmologique, la plus ancienne lumière encore présente dans l'Univers, et Kao a mis au point la transmission de la lumière dans les fibres pour la communication optique.

Autant de raisons de choisir 2015 pour commémorer l'ensemble de ces avancées majeures.

\section{Qu'est-ce que la « photonique »?}

La coordination et l'animation de l'Année internationale de la lumière en France ont été prises en charge par le Comité national d'optique et de photonique (CNOP).

La photonique est l'étude de composants permettant la génération, la transmission, le traitement ou la conversion de signaux optiques. Elle étudie donc les photons comme onde ou comme corpuscules et s'étend de l'ultraviolet à l'infrarouge, avec des applications qui résident principalement dans le domaine du spectre visible.

En s'associant avec l'électronique et l'informatique, l'optique est devenue "photonique » et un champ immense d'applications s'est ainsi ouvert. La maîtrise de l'ultraviolet et de l'infrarouge a permis à l'homme d'élargir considérablement son domaine de vision. Inventé en 1960, le laser a apporté une révolution (voir Reflets de la physique, $\mathrm{n}^{\circ} 21$, octobre 2010).

L'optique est maintenant présente dans tous les domaines de notre vie quotidienne, comme dans les techniques de pointe: sécurité, thermographie industrielle et médicale, contrôle de la pollution, analyse et contrôle non destructif, fibre optique, etc. L'optique photonique est au cœur de l'innovation. Elle est présente dans tous les corps de métiers et est indispensable à un développement harmonieux de nos technologies. La photonique est d'ailleurs reconnue au niveau européen comme l'une des six technologies clés.

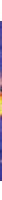




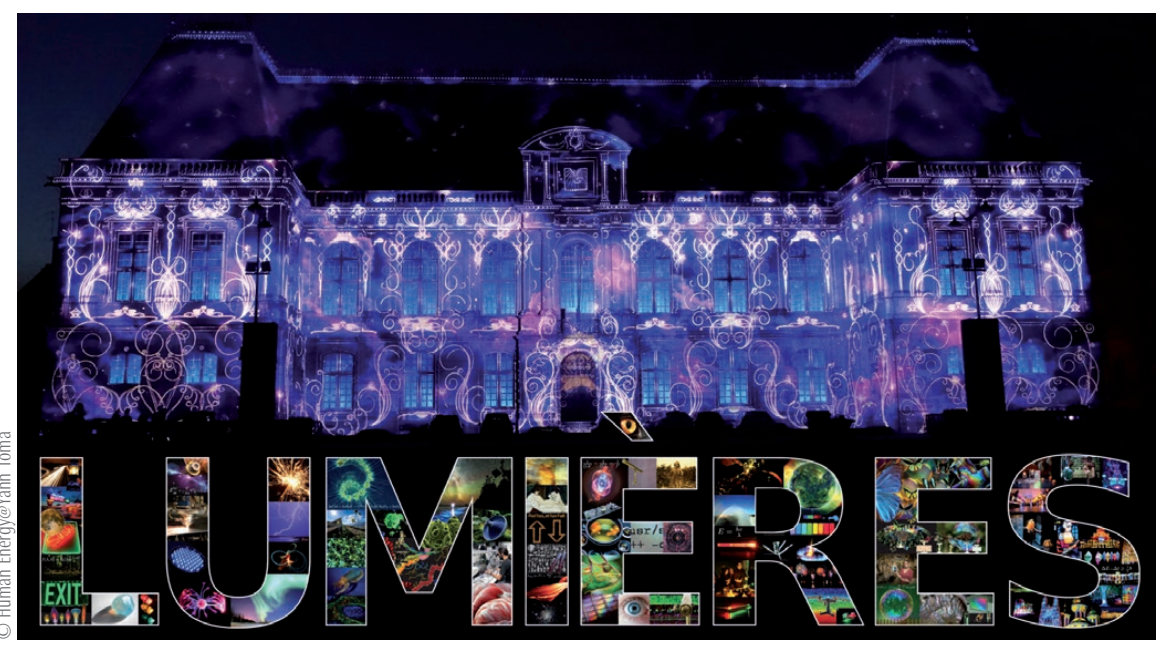

3. Illumination du Parlement de Bretagne à Rennes en juillet-août 2015. Réalisation : Spectaculaires Allumeurs d'images - Université de Rennes 1.

4. « Human Energy », de l'artiste français Yann Toma, transforme la tour Eiffel en une immense antenne énergétique, pour affirmer l'état d'urgence climatique.

\section{\〉>}

\section{Lumière, vie quotidienne et culture}

La ligne directrice du Comité national a consisté à promouvoir notre héritage culturel et à mettre en relation les aspects scientifiques, artistiques et culturels de la lumière à travers de nombreuses manifestations dans toute la France. Nous avons montré la place qu'occupe la lumière dans notre vie quotidienne et avons promu ses capacités à apporter des solutions aux grands défis contemporains que sont l'énergie, l'éducation, l'agriculture et la santé, et à s'intégrer dans une démarche de développement durable. En quelques décennies, les avancées technologiques ont crû de façon exponentielle ; elles alimentent des travaux de recherche sur les sources de lumière, les émissions spectrales et les matériaux. On assiste à un essor industriel pour recréer la lumière et mettre au point des éclairages performants, diversifiés et confortables. L'apparition des sources à LED révolutionne totalement les techniques de l'éclairage, et la notion d' "éclairage intelligent » s'impose comme une évidence. Cette révolution technologique associant lumière électronique et données numériques nous conduit vers une autre représentation du monde : une nouvelle dimension culturelle où la lumière fusionne volontiers avec la matière sur nos écrans, mais aussi avec toutes sortes de supports où des signaux et images de lumière s'animent et s'échangent en temps réel.

Jusqu'au XIX ${ }^{\mathrm{e}}$ siècle, la culture n'était pas dissociée des sciences, cette séparation est récente. L'Année de la lumière promeut une approche pluridisciplinaire : plusieurs colloques croisent les arts et les sciences, par exemple "Les Lumières de la vie " à l'université Paris Diderot, un atelier à la Saline royale d'Arc-et-Senans, les journées de l'optique à Nice, le colloque organisé à Paris avec Gérard Mourou (École polytechnique) et Monica Preti (musée du Louvre) en novembre 2015, sur les découvertes de Fresnel et sur les évolutions, au siècle suivant, de la représentation de la lumière en peinture. Une remarquable exposition au Mudam à Luxembourg, "Eppur si muove " ("Et pourtant elle tourne ») fait dialoguer des objets scientifiques sortis des réserves du CNAM avec des œuvres contemporaines. Une autre, "L'œil phénomène ", à la Fondation Vasarely, questionne la perception visuelle, avec des œuvres de Michel Paysant ; cet artiste dessine à l'échelle nanométrique, ses œuvres ne peuvent être vues qu'à l'aide d'un microscope électronique à balayage.

Lors du Congrès OPTIQUE 2015, le parlement de Bretagne à Rennes a été habillé de lumières (fig. 3), offrant tous les soirs, du 8 juillet au 23 août 2015, un voyage poétique et ludique au cœur de la capitale bretonne. En décembre 2015, a été présentée à la tour Eiffel la grande réalisation artistique participative "Human Energy”, de l'artiste et chercheur Yann Toma (fig. 4).

Costel Subran Président du Comité national de l'Année de la Lumière en France

\section{Comité national}

Parrainé par les prix Nobel de physique

Claude Cohen-Tannoudji et Serge Haroche

\section{Bureau exécutif}

Costel Subran, président du Comité national

Éric Lambouroud, responsable de la communication

Ivan Testart, responsable sponsoring et trésorier

Agnès Henri, secrétaire

Catherine Herce, responsable du site Internet

Vice-Presidents

John Dudley - FEMTO-ST, Chairman de I'IYL2015

Étienne Klein - CEA

Michèle Leduc - IFRAF-ENS-CNRS

Gérard Mourou - École polytechnique

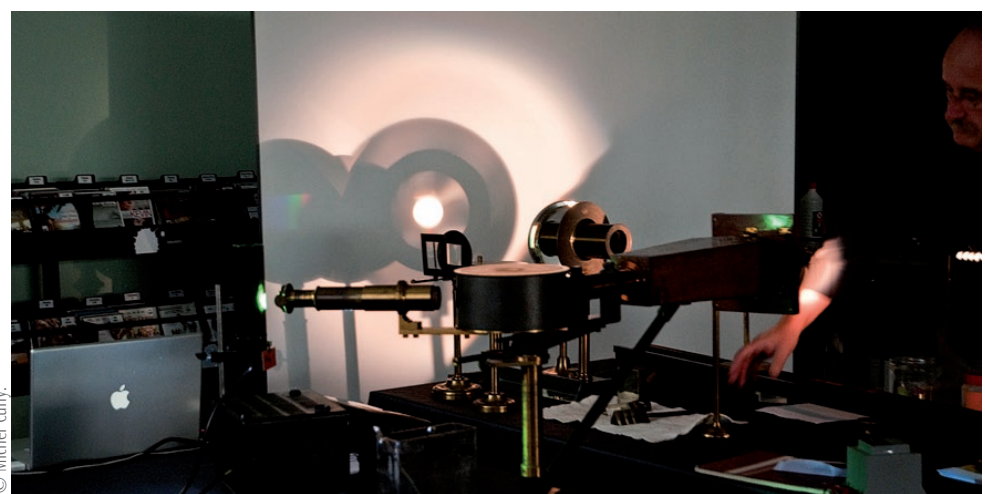

Atelier de physique « Mirages, arc-en-ciel, spectres ", à l'Université de Lyon 1. 